\title{
Mechanical Reinforcement of Electrospun Poly(Vinyl Alcohol) by a-FeOOH Nanowires
}

\author{
Anna Sutka, ${ }^{1,2}$ Martin Jarvekulg, $\quad 3,4$ Andris Sutka, ${ }^{4,5}$ Ivo Heinmaa, ${ }^{6} \quad$ Uno Maeorg, Krisjanis Smits, ${ }^{7}$ \\ Martin Timusk 4,9 \\ ${ }^{1}$ Institute of Design Technology, Riga Technical University, Kipsalas 6, Riga LV-1048, Latvia \\ ${ }^{2}$ Latvian State Institute of Wood Chemistry, Dzerbenes 27, Riga LV-1006, Latvia \\ ${ }^{3}$ Biomaterials Research Laboratory, Riga Technical University, Paula Valdena 3/7, Riga LV-1048, Latvia \\ ${ }^{4}$ Institute of Physics, University of Tartu, W. Ostwaldi 1, Tartu 50411, Estonia \\ ${ }^{5}$ Institute of Silicate Materials, Riga Technical University, Azenes 14/24, Riga LV-1048, Latvia \\ ${ }^{6}$ National Institute of Chemical Physics and Biophysics, Akadeemia 23, Tallinn 12618, Estonia \\ ${ }^{7}$ Institute of Chemistry, University of Tartu, Ravila 14a, Tartu 50411, Estonia \\ ${ }^{8}$ Institute of Solid State Physics, University of Latvia, Kengaraga 8, Riga LV-1063, Latvia \\ ${ }^{9}$ Institute of Technical Physics, Riga Technical University, Paula Valdena 3/7, Riga LV- 1048, Latvia
}

We report the mechanical performance of a-FeOOH nanowire reinforced poly(vinyl alcohol) (PVA) compos-ite nanofiber mat, fabricated using straightforward aqueous processing methods. Goethite $(\mathrm{a}-\mathrm{FeOOH})$ nanocrystals have a high elastic modulus and $-\mathrm{OH}$ rich surface, ensuring strong interactions with hydrophilic polymers and effective reinforcement. Needle-less electrospinning resulted in alignment of the nanowires along fibre axis, as confirmed by transmittance elec-tron microscopy studies. Produced composite PVA nanofibers containing 10 wt\% goethite nanoparticles exhibited an outstanding fivefold increase in Young's modulus and 2.5-fold improvement of tensile strength compared to mats of neat PVA. The addition of a$\mathrm{FeOOH}$ had a significant influence on glass transition temperature indicating formation of interphase regions around nanowire inclusions. Observed properties are explained by nanowire grafting in the precursor

Correspondence to: A. Sutka; e-mail: anna.sutka@rtu.lv Contract grant sponsor: financial support of the Estonian Research Council; contract grant numbers: PUT1096, PUTJD578; contract grant sponsor: Institutional Research Funding Projects; contract grant numbers: IUT20-17, IUT23-7. solution, extensive interactions between the adsorbed PVA chains and the matrix and percolation of inter-phase regions at $10 \mathrm{wt} \%$ a-FeOOH.

\section{INTRODUCTION}

Goethite (a-FeOOH) is the most abundant crystalline iron mineral in nature [1]. Nanowires of a-FeOOH occur as the inorganic component in the strongest known natu-ral material where the aligned particles provide the com-posite with extreme mechanical properties [2]. In addition to a high elastic modulus (187 GPa [3]), the surface of the goethite crystals presents a highly dense coating of $-\mathrm{OH}$ groups [4] to the polymer matrix. This effectively promotes mechanical reinforcement of the polymer through strong attractive nanoparticle-matrix interactions with hydrophilic moieties on the polymer chains, due to robust hydrogen bonding [5-7], resulting in the formation of an extensive interphase. In addition to improved mechanical properties, a-FeOOH may provide polymer materials with other functionalities. Goethite is reported to be narrow band gap semiconductor with catalytic 
activity for Photo-Fenton reactions [8, 9] and room temperature ferromagnetism [9] and birefringent properties in polymer matrices [10]. Also, strong absorption of visible light by goethite might produce radicals that could initiate polymerization of monomer adsorbed at the surface of goethite nanoparticles [10].

The main aim of the present work is to combine the strong interactions between electrospun PVA nanofibers and goethite nanowires with directional co-alignment of the reinforcing mineral fillers for superior mechanical reinforcement. Poly(vinyl alcohol) (PVA) is a hydrophilic polymer with excellent chemical properties, biodegrability, low cost and can be easily electrospun to nanofibers with applications in filtering, reinforcing materials, wound dressing, tissue engineering scaffolds and controlled drug delivery systems [11]. However, moderate mechanical integrity and moisture sensitivity of the virgin polymer limit its uses [12]. A variety of approaches that have been developed to improve the properties of PVA have been reviewed by several authors [13-15]. One of the most promising methods, modification of PVA by nanoparticle additives has gained increasing attention [5, 14, 16-25]. In general, the significant enhancements observed in nanoparticle-reinforced polymers are attributed to an interphase, a region surrounding the inclusions where the properties differ from the bulk. This phenomena that occurs in on the surface of the particle and its vicinity, as well as, its influence nano-composites has therefore received considerable attention in both experimental [17, 21, 26, 27] and theoretical work or modeling [28-41]. The size, shape and dispersion state of the particles as well as interactions on nanoparticle-polymer interface have been found to be critical in maximizing the desired influence of additive [27, $31,32,34,37,38,41,42]$. In this work, we demonstrate extensive hydrogen bonding between PVA and goethite nanowires from a "chimie douce" synthesis and the resulting remarkable reinforce-ment in electrospun PVA nanocomposite fibers.

High aspect ratio fillers (nanowires) in polymers by themselves can have more significant influence on mechanical properties in comparison with low aspect ratio fillers.

Chemin et al studied polymer-ferric oxide composite systems and found that if dispersed particles are oriented randomly, then the magnitude of reinforcing effect correlates to the specific surface area of the mineral and is independent from particle geometry [10]. However, in the case where oblong particles are dispersed in the polymer in an ordered manner, the aspect ratio and orientation state can have a significant effect on the mechanical prop-erties of polymer based composites [34, 43-45] where the alignment of the additive particles can be induced by imposing flow on the precursor suspension [46]. Among other processing methods, electrospinning can be applied to achieve orientation of uniaxial additive particles in polymer due to shear forces acting on the material during fiber formation [8, 43, 44, 46-49]. In the experiments presented here, the high aspect ratio goethite nanowires exhibited directional alignment in electrospun composites, resulting in increased reinforcement.

\section{EXPERIMENTAL}

Goethite (a-FeOOH) nanowires were synthesized by straightforward and environmentally friendly "chimie douce" precipitation method at room temperature. In a typical procedure $\mathrm{Fe}\left(\mathrm{NO}_{3}\right)_{3} \mathrm{9H}_{2} \mathrm{O}$ (98\%, Sigma Aldrich) was dissolved in milli-Q water to obtain $0.1 \mathrm{M}$ solution. Simultaneously, $1 \mathrm{M}$ aqueous solution of $\mathrm{NaOH}(98 \%$, Sigma Aldrich) was prepared. In the next step, both solutions were mixed together in equimolar ratio) and left to stir for one hour. The mixture was then transferred into a closed glass bottle and kept in the dark for $72 \mathrm{~h}$. The obtained precipitates were centrifuged and washed by milli$\mathrm{Q}$ water several times but not dried in order to pre-vent nanowire agglomeration. Obtained a-FeOOH nano-wires were dispersed in milli-Q water by ultrasonic treatment for $10 \mathrm{~min}$ (ultrasonic processor UP200H, fre-quency $24 \mathrm{kHz}$, sonotrode S3, Ø $3 \mathrm{~mm}$ ) and PVA (Sigma Aldrich) with molecular weight of $145,000 \mathrm{~g} / \mathrm{mol}$ and degree of hydrolysis 99.0-99.8 mol\% was then added at desired concentration by magnetic stirring at $808 \mathrm{C}$.

Electrospinning was carried out at room temperature (228C) and at a relative humidity of $30 \% 62 \%$ on needleless high-voltage Nanospider ${ }^{\text {TM }}$ NS Lab 200 (Elmarco) equipped with cylindrical spinning electrode. Produced fiber was collected on an antistatic nonwoven polypropylene material (surface density Q $521.563 \mathrm{~g} \mathrm{~m}^{22}$ ). Sixty to sixty-five $\mathrm{kV}$ DC voltage was applied to an electrode of 2 $\mathrm{cm}$ diameter and $15 \mathrm{~cm}$ length electrode rotated at $4 \mathrm{rpm}$. The distance between the spinning and the collect-ing electrodes was kept at approximately $15 \mathrm{~cm}$.

The microstructural features of the samples were studied by scanning electron microscopy (SEM, Helios Nanolab, FEI) and transmission electron microscope (TEM, Tecnai G20, FEI) operated at $200 \mathrm{kV}$. The average diameters were evaluated by measuring around hundred fibre diameters from SEM images. The crystal phases of the synthesized a-FeOOH nanowires and PVA/a-FeOOH composites were characterized by powder X-ray diffraction (XRD) recorded from 158 to 808 at a scanning rate of 18/min using an Ultima1 X-ray diffractometer (Rigaku, Tokyo, Japan) with $\mathrm{Cu} \mathrm{Ka}$ radiation.

Attenuated total reflection-Fourier transform infrared (ATR-FTIR) spectroscopy was used for additional studies of the samples. All samples were recorded on a Spectrum One (Perkin Elmer, UK) FTIR spectrometer in the range of $4,000-600 \mathrm{~cm}^{21}$ at a resolution of $4 \mathrm{~cm}^{21}$ after 32 continuous scans. The ATR top plates have composite zinc selenide ( $\mathrm{ZnSe})$ and diamond crystals.

The interaction between PVA and a-FeOOH nano-wires was studied by obtaining solid state carbon ${ }^{13} \mathrm{C}$ CP-MAS NMR spectra. The spectra were recorded with Bruker AVANCE-II-600 spectrometer at 14.1T magnetic 


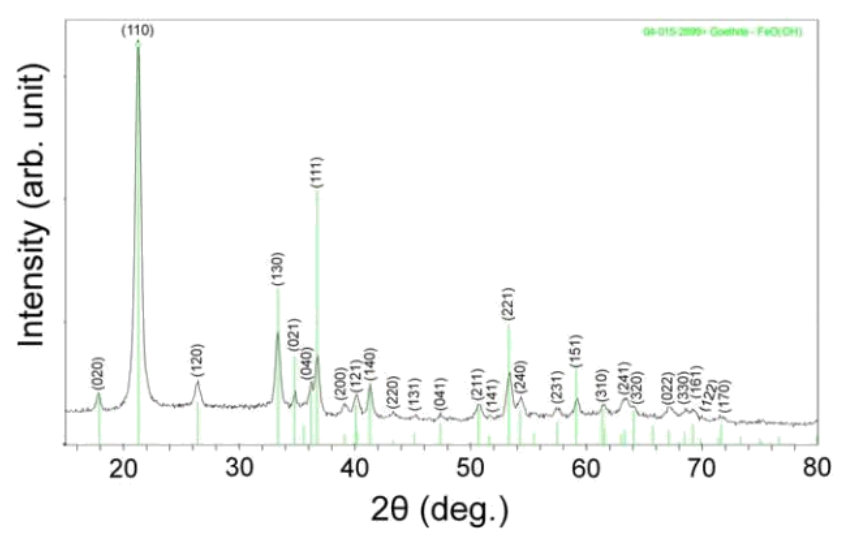

FIG. 1. X-ray diffraction pattern of synthesized goethite nanowires. [Color figure can be viewed at wileyonlinelibrary.com]

field using home built MAS probe for $4 \mathrm{~mm}$ zirconia rotors at $12.5 \mathrm{kHz}$ sample spinning speed. In ordinary pulse sequence after $0.7 \mathrm{~ms}$ ramped cross polarization pulse the signal was registered in the presence of swTPPM sequence for proton decoupling. 5s relaxation delay was used between accumulations. The intensity in the spectra was normalized to the weight of the sample and to the number of accumulations.

The thermal properties of PVA/a-FeOOH composites were studied via differential scanning calorimetry (DSC, 822e METTLER TOLEDO). The parameters were as follows: $8 \mathrm{mg}$ sample weight, $258 \mathrm{C}$ to $2408 \mathrm{C}$ with $108 \mathrm{C} / \mathrm{min}$ heating and $308 \mathrm{C} / \mathrm{min}$ cooling rates.

The room-temperature mechanical properties of the electrospun composite nanomats were measured by Ins-tron Universal Tester (Model 2519-107) with Bluehill software, applying a deformation speed of $1 \mathrm{~mm} / \mathrm{min}$. Samples 20 $\mathrm{mm}$ in length and $10 \mathrm{~mm}$ in width were cut from the central part of the composite nanomats to provide a similar thickness. The average thickness for electrospun PVA and PVA/a-FeOOH nanofiber composite mats was around $100 \mathrm{~lm}$.

\section{RESULTS AND DISCUSSION}

The XRD pattern of the obtained a-FeOOH nanowires is shown in Fig. 1. The precipitation synthesis yielded phase pure goethite a-FeOOH (ICDD 04-015-2899) with orthorhombic phase (space group Pbnm, No. 62) having lattice constants of a $54.6158 \mathrm{~A}, \quad$ b $5 \quad 9.9545 \mathrm{~A}$, and C $53.0233 \mathrm{~A}$. Although obtained at room temperature, the sharp diffraction peaks of the a-FeOOH nanowires indicate well-crystallized material. No peaks arising from impurities were detected by XRD.

The microstructural features of the synthesized aFeOOH were studied by TEM (Fig. 2). The goethite nanocrystals have anisotropic shape with length around 500 $\mathrm{nm}$, diameter from 20 to $150 \mathrm{~nm}$ and aspect ratio up to 30 . TEM images show that the majority of nanowires are grouped in bundles of two or three (Fig. 2a). The diameter of single nanowire is around $2165.5 \mathrm{~nm}$. HR-TEM images (Fig. 2b) demonstrate high crystallinity of the synthesized a-FeOOH nanowires with evident highly ordered crystal lattice. The a-FeOOH nanowire formation is attributed to olation of tetrameric polycation species to embryos of double chains of octahedra, which is a charac-teristic of the structure of goethite [50]. A subsequent connection between chains by oxolation leads goethite nanowire growth [50]. This process occurs at $\mathrm{pH}$ values higher than 8. In our synthesis $\mathrm{pH}$ was $>13$.

The morphology of PVA and composite PVA/FeOOH nanofiber mats is presented in Fig. 3. All produced mats are formed of continuous, uniform, bead-free fiber. The aver-age diameter is slightly decreased (from $0.50660 .164 \mathrm{~mm}$ to $0.36160 .104 \mathrm{~mm}$ ) if a-FeOOH nanowires are added.
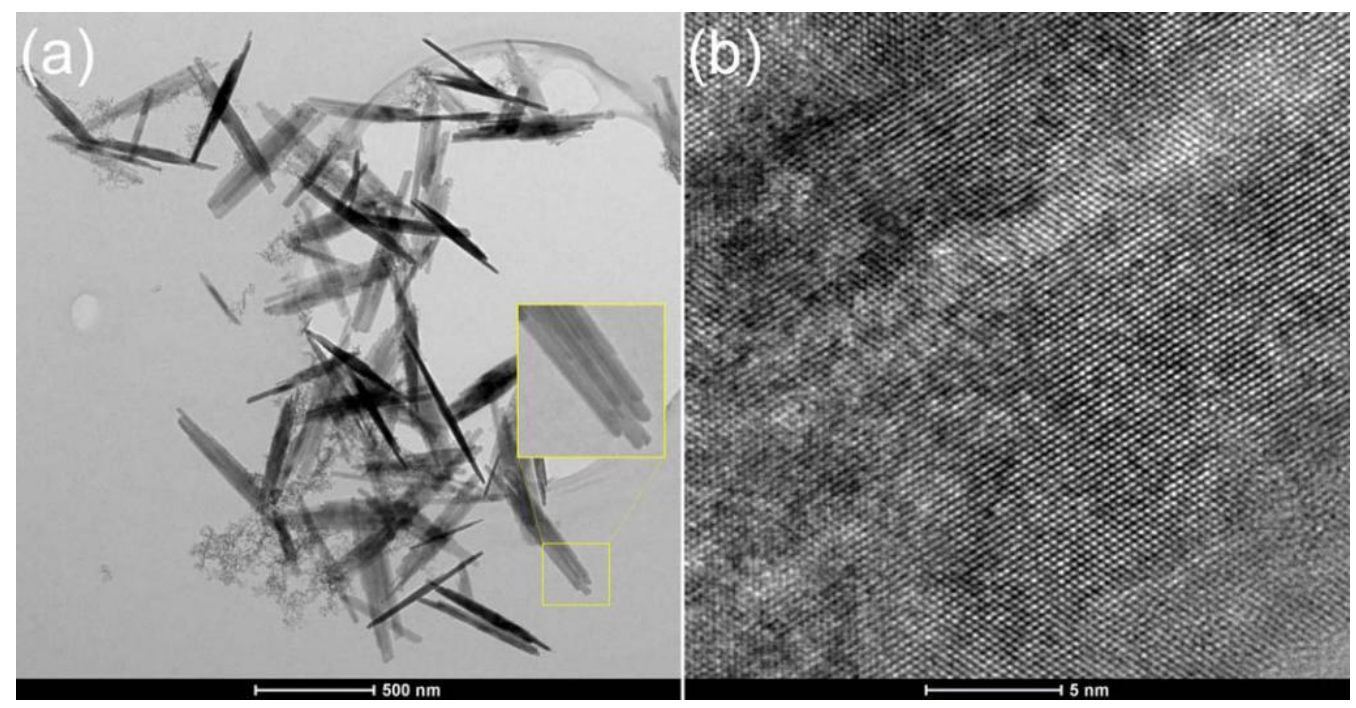

FIG. 2. TEM micrographs of synthesized mineral particles. The morphology of goethite nanowires (a) and high resolution image demonstrating high crystallinity of the material (b). [Color figure can be viewed at wileyonlinelibrary.com] 

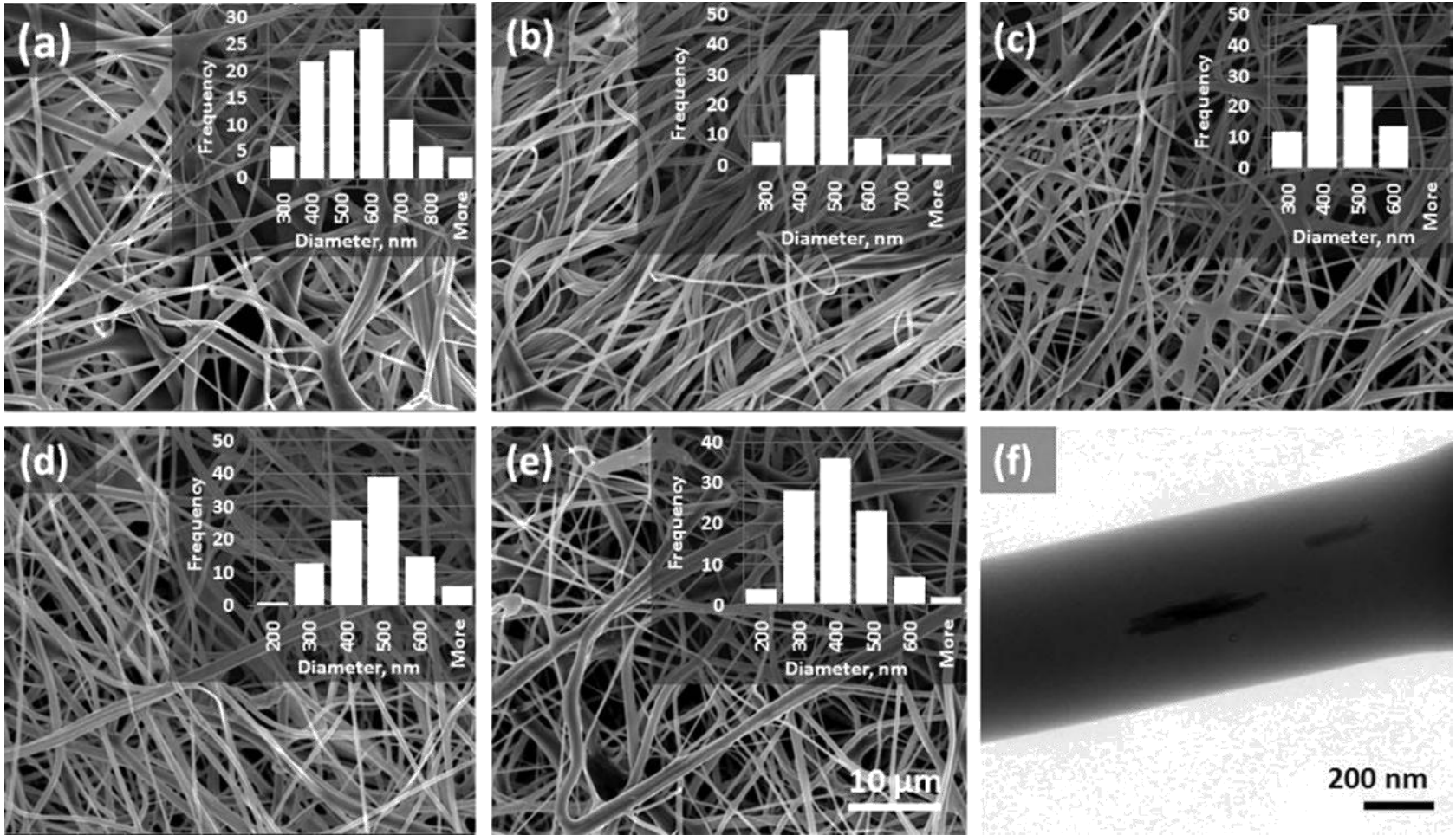

FIG. 3. SEM images of electrospun PVA nanofibers reinforced by a-FeOOH nanowires at different concentrations: (a) $0 \mathrm{wt} \%$, (b) $1 \mathrm{wt} \%$, (c) $2.5 \mathrm{wt} \%$, (d) $5 \mathrm{wt} \%$, (e) $10 \mathrm{wt} \%$. Panel (f) presents TEM image of effec-tive a-FeOOH nanowire incorporation into the reinforced PVA nanofiber.

This kind of behavior has been widely reported in case of electrospun composite nanofibers [22], where some poly-mer fraction is replaced by modifying filler. The average diameters of fibers containing $0,1,2.5,5$ and $10 \mathrm{wt} \%$ of goethite were $0.50660 .164,0.44760 .121,0.39760 .078,0.41160 .109$ and $0.36160 .104 \mathrm{~mm}$, respectively. Figure 3f shows TEM image of PVA nanofibers containing $5 \mathrm{wt} \%$ of a-FeOOH nanowires. Goethite nanowires are well incor-porated into the PVA nanofiber. After careful examination by TEM, we found out that goethite nanowires are homoge-neously dispersed through PVA matrix. As expected, the a-FeOOH nanowires were also aligned parallel to the axis of PVA fiber. Electrospun polymer nanofibers are stretched in electrical field [51]. Resulting shear causes nanowire orien-tation in the direction of pulling that coincides with the fiber axis. During the elongation of polymer jet, macromo-lecules orient in the direction of stretching and also exert a co-aligning force on the nanowires [43, 52].

To further validate the incorporation of a-FeOOH into the electrospun PVA fibers, the mats were characterized by XRD and FTIR. The XRD graphs for neat PVA fibers and PVA/a-FeOOH composites are shown in Fig. 4. The XRD graphs show a peak to PVA at around 208. In case of PVA/a-FeOOH composites the characteristic peaks of goethite crystal phase are also clearly observable, indicating presence of a-FeOOH in nanofiber mats.

Figure 5 shows the ATR-FTIR absorbance spectra of electrospun PVA fibers, a-FeOOH nanowires and PVA/ a$\mathrm{FeOOH}$ composite fibers in the range of 4,000
$600 \mathrm{~cm}^{21}$. The goethite ATR-FTIR spectrum agrees with literature data, with a strong intensity band at $884 \mathrm{~cm}^{21}$ and $790 \mathrm{~cm}^{21}$ caused by $\mathrm{d}(\mathrm{O}-\mathrm{H})$ deformation vibrations (in plane and out of-plane bending) and a broad band at 3,133 $\mathrm{cm}^{21}$ due to $\mathrm{O}-\mathrm{H}$ stretching vibrations [53-58]. The maximum of the band at $893 \mathrm{~cm}^{21}$ and $792 \mathrm{~cm}^{21}$ increased with the increasing content of goethite nano-wires into the PVA matrix suggesting a-FeOOH incorpo-ration into the mat. However, the spectra give no information on bond formation between PVA and the mineral particles.

NMR studies were also conducted in order to detect possible covalent bonding between the a-FeOOH particles

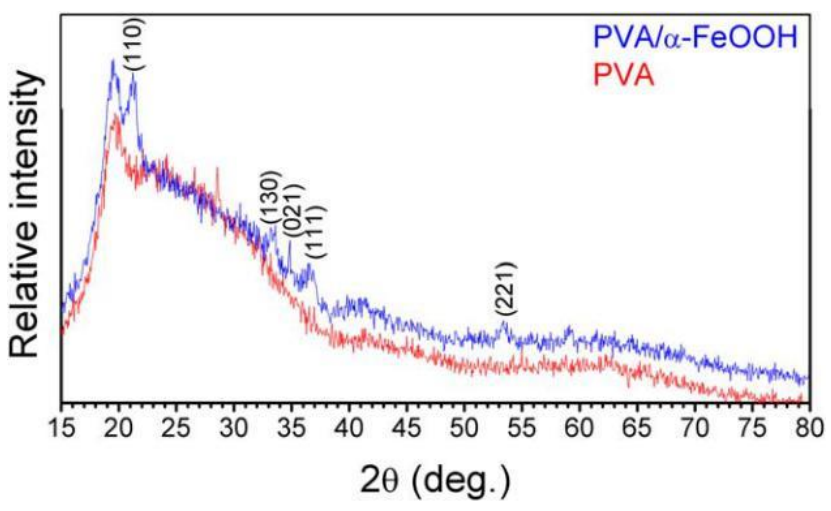

FIG. 4. XRD graphs of electrospun PVA and PVA/a-FeOOH nanofib-ers. [Color figure can be viewed at wileyonlinelibrary.com] 


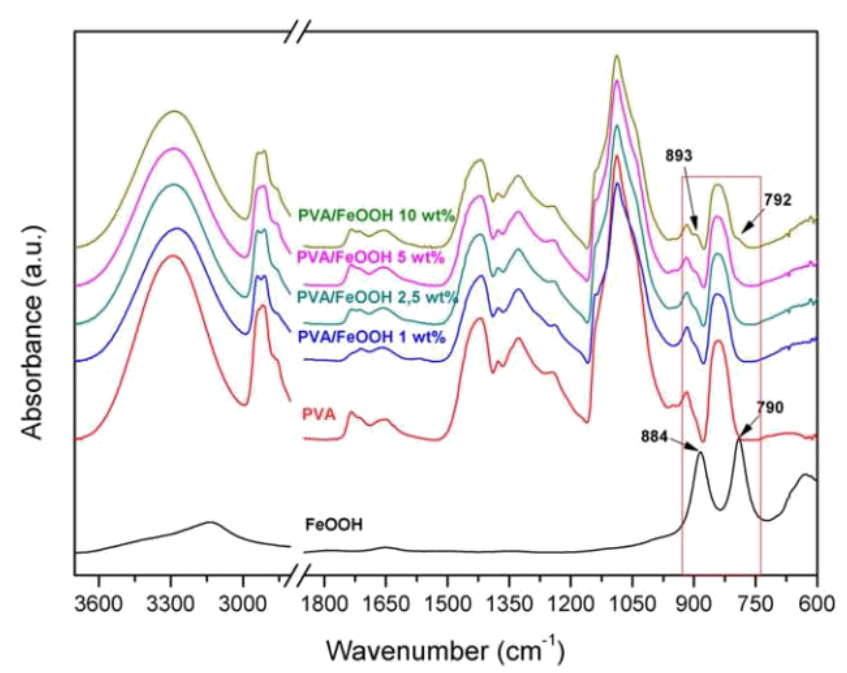

FIG. 5. ATR-FTIR spectra for a-FeOOH nanowires, electrospun PVA fibers and PVA/a-FeOOH composite fibers. [Color figure can be viewed at wileyonlinelibrary.com]

and PVA. CP-MAS ${ }^{13} \mathrm{C}$ NMR spectra of different aFeOOH reinforced PVA samples are shown in Fig. 6. The spectra of PVA consist of $\mathrm{CH}_{2}$ carbons line at $46 \mathrm{ppm}$ and of the lines I, II, and III (at 76.3, 70.6 and 65.1 ppm) belonging to $\mathrm{CH}$ carbons in conformations with two-, oneand zero intramolecular hydrogen bonds in the triad sequences, respectively $[59,60]$. In addition, a weak methyl line (chain ends) at $22.5 \mathrm{ppm}$ can be seen. All samples of different a-FeOOH content show almost iden-tical spectra. The width of the lines increases gradually with increasing a-FeOOH content by $0.2-0.3 \mathrm{ppm}$ only. The biggest effect to the spectra is a gradual loss of line intensities. This phenomenon is caused by the magnetic moment of iron $\mathrm{Fe}^{31}$ ions. These magnetic moments pro-duce large magnetic field at nearest carbons which wipes out their signal from ${ }^{13} \mathrm{C}$ NMR spectrum. Linear decrease of the signal intensity indicates that a-FeOOH concentra-tion is small enough that the wipeout effect of neighbor-ing particles does not overlap. Strong wipeout effect shows that the a-FeOOH particles are tightly packed into the PVA matrix and can thus be taken as a sign of an interphase region, i.e., a restructured polymer layer strongly adhered to the additive particles [10].

DSC curves for second heating cycle for neat PVA and PVA/a-FeOOH composite samples are depicted in Fig. 7a. The small variation in the temperatures of endo-thermic transition melting temperatures indicates that a-FeOOH addition does not have significant influence on the degree of polymer crystallinity. However, the mid-point of glasstransition occurring from 55 to $908 \mathrm{C}$ shifts to higher temperatures (Fig. 7b) increasing from $698 \mathrm{C}$ to $818 \mathrm{C}$ in case of neat PVA and $10 \mathrm{wt} \%$ of goethite, accordingly. A shift in glass-transition temperature is rou-tinely observed in nanoparticle-polymer composites, including PVA-based materials $[14,17,19]$ and has been directly linked to the formation of glassy interphase regions. Furthermore, the magnitude of the change in glasstransition temperature has been found to increase with increased interphase polymer volume fraction [36, 61]. It can be thus speculated that the logarithmic nature of dependence between glass-transition temperature and goethite content indicates percolation of interphase regions, i.e., the composite becomes gradually saturated with interphase.

As expected, a-FeOOH nanowire addition results in a remarkable improvement in the mechanical strength of electrospun PVA fibers. Figure 8 shows up to fivefold increase in Young's modulus and 2.5-fold increase in the tensile strength at up to $10 \mathrm{wt} \%$ a-FeOOH concentration. The average Young's modulus increased from 0.0760 .008 $\mathrm{GPa}$ to $0.3760 .023 \mathrm{GPa}$ suggesting strong interactions and effective stress transfer between goethite nanowires and the PVA matrix. Since spectroscopy meth-ods indicated no significant changes in covalent bonding or molecular ordering, the immediate interaction between the polymer and mineral surface can be attributed to hydrogen bonding and possible coordinate bond forma-tion. Kavanagh et al studied the adsorption of PVA on goethite particles [46]. They found that the amount of adsorbed PVA greatly exceeds a close packed monolayer and proposed that the free ends of adsorbed polymer chains are available for inter-particle bonding. In the pre-sent case, the volume fraction of a-FeOOH is below $5 \mathrm{wt} \%$, moreover the nanowires are dispersed homoge-neously as bundles (Fig. 3f) and thus this kind of direct bridging between particles is unlikely. Nevertheless, the free ends of adsorbed PVA chains can participate in fur-ther polymer-polymer interactions. A similar situation can be imagined in case of coordinate bonding. PVA, a 1,3-diol, is known to form stable complexes with iron(III). ${ }^{30}$ The polymer is atactic so not all the hydroxyl groups can participate in coordinate bonding and remain available for

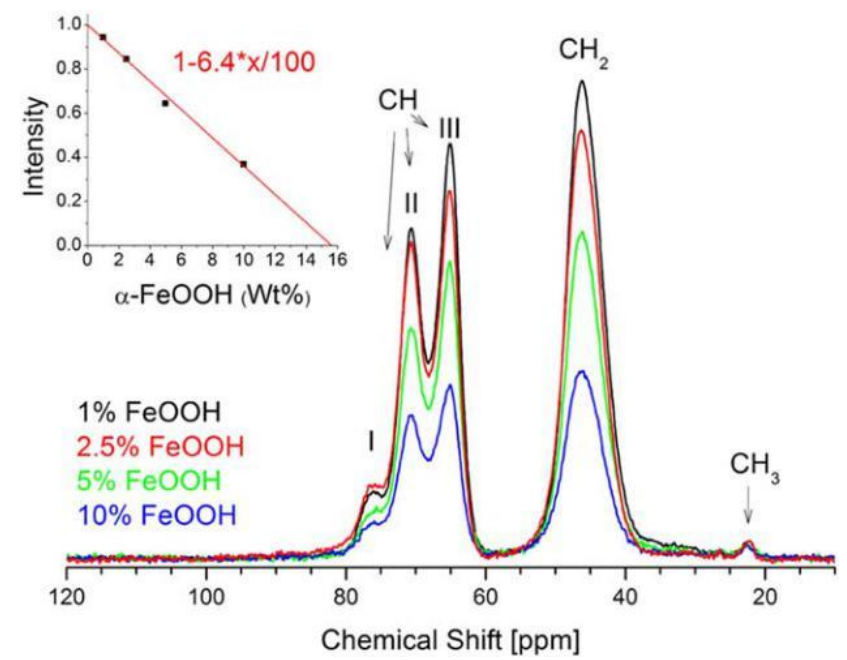

FIG. 6. 13C CP-MAS NMR spectra of PVA nano-fibers reinforced by $1 \%$, $2,5 \%, 5 \%$ and $10 \%$ a-FeOOH. Inset shows intensity of $\mathrm{CH} 2$ line as a function of a-FeOOH content. [Color figure can be viewed at wileyonlinelibrary.com] 

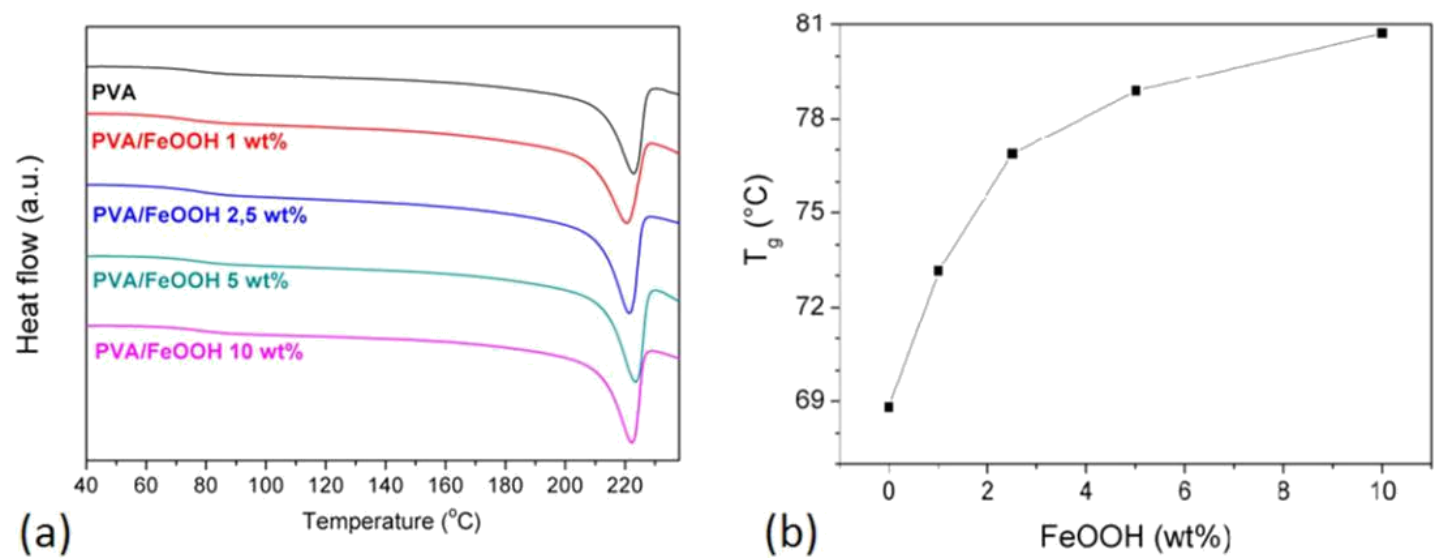

FIG. 7. (a) The second heating cycle DSC curves for samples loaded with the different contents of a-FeOOH; (b) the variation of glass transition temperatures with goethite nanowire content. [Color figure can be viewed at wileyonlinelibrary.com]

further interactions. This kind of spontaneous grafting of particles is likely to result in steric stabilization in the aqueous precursor solution [32] and leads to strong particle embedment through entanglements between adsorbed chains and surrounding polymer in dry fibers $[17,33,39$, 62]. The elasticity asymptotically reaches a maximum value at around $10 \mathrm{wt} \%$ nanowire content. The leveling and subsequent reversed influence of increased filler concentration is often explained with particle agglomeration
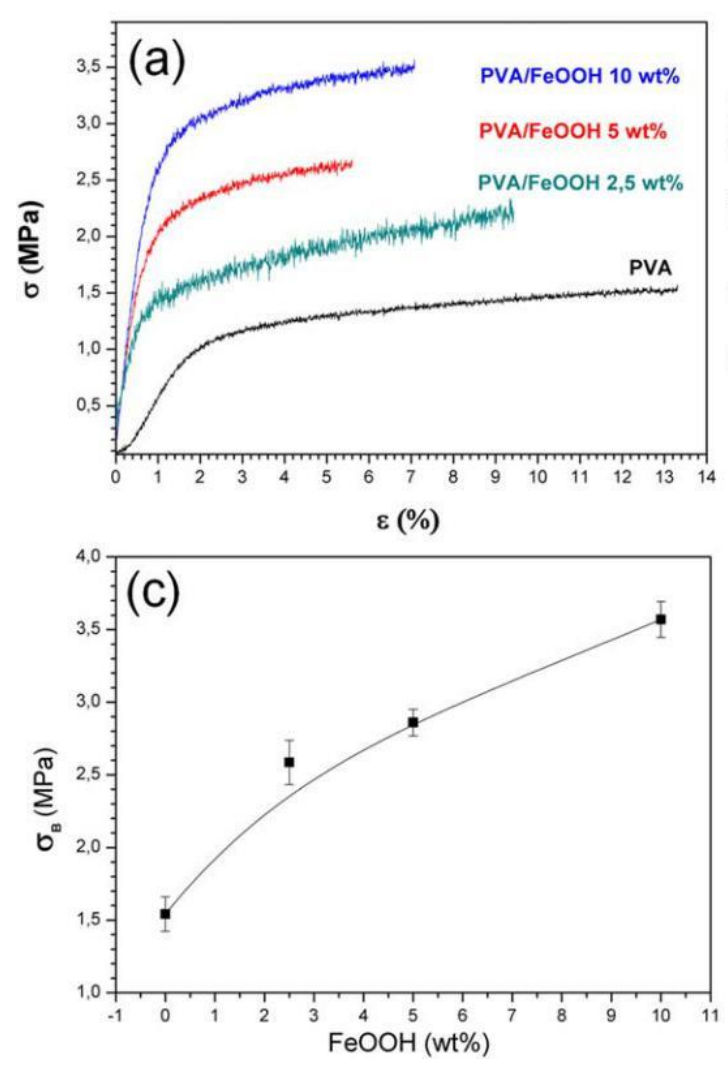

[16]. In principle, inhomogeneity, i.e., particle-rich domains can also occur in case of grafted particles [63]. In the latter case the influence of aggregates can be expected to be different as there is no immediate contact between the mineral particle surfaces and the mechanical properties are determined by the contacts between inter-phase domains [29]. However, the fact that electrospin-ning led to uniform fibers at up to $10 \mathrm{wt} \%$ nanowire concentrations indicates that there was no significant
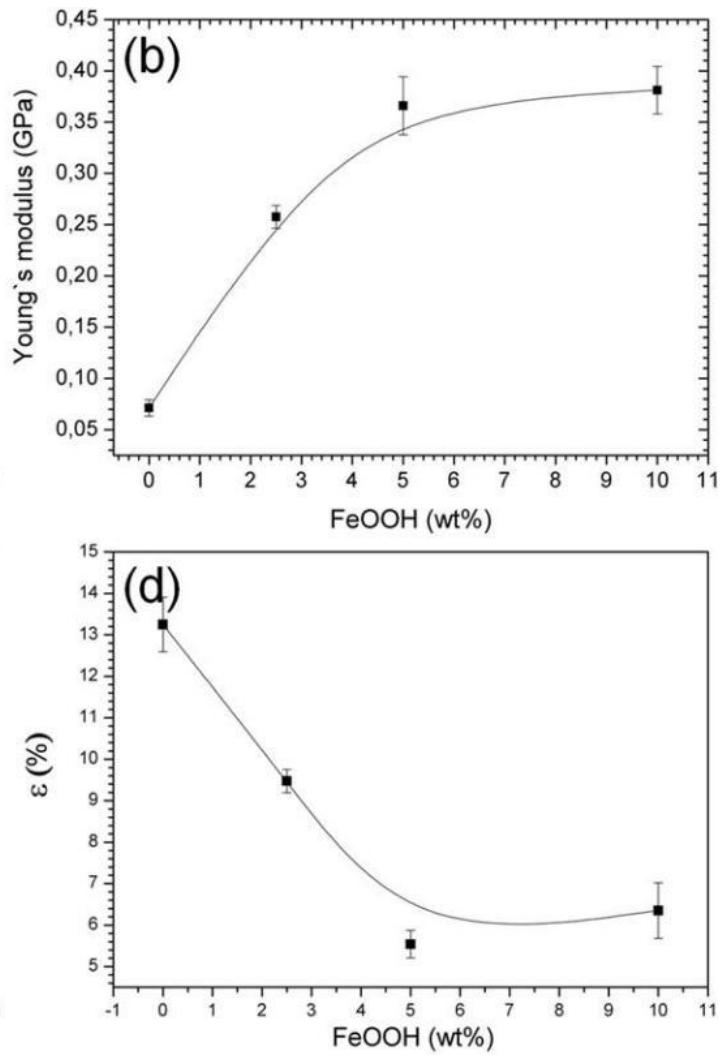

FIG. 8. Mechanical properties for electrospun PVA fibers and PVA/a-FeOOH composite fibers with differ-ent a-FeOOH content: (a) stress-strain curves; (b) Youngs modulus (GPa); (c) stress at break (rB, MPa) and (d) extension at break $(\mathrm{e}, \%)$. [Color figure can be viewed at wileyonlinelibrary.com] 
fluctuation in solution viscosity during jet formation, thus the occurrence of any aggregates (larger than the nano-wire bundles) is not likely. Therefore, it can speculated that at 10 wt\% is sufficient to effectively influence poly-mer configuration in the majority of the volume and result in continuous network of interphase domains throughout the fiber [35] whereas further increase in filler concentration would lead to gradual overlapping or inter-ference between the interphase shells [17].

Mechanical strength of electrospun fiber mats also depends on the interfiber binding at intersections, thus the mats with smaller fiber diameters have a higher density of bonding contacts between fibers [64]. Smaller diameter fibers can exhibit size dependent mechanical properties due to the so-called size dependent surface effect [65]. High Young's modulus and strong size dependence for neat electrospun PVA nanofibers, reaching values up to 500 $\mathrm{GPa}$ for fibers with sizes lower than $20 \mathrm{~nm}$, was demonstrated by $\mathrm{Fu}$ et al., where authors used the nanoscale three-point bending tests to characterize mechanical properties [66]. However, as noted above, the addition of a$\mathrm{FeOOH}$ has almost no influence on the fiber diameter distribution. Also, for electrospun PVA nanofibers a significant size effect should appear for only sizes lower than $300 \mathrm{~nm}$ [65] and all of the samples were found to contain an insignificant number of fine fibers. Moreover, the composition containing $5 \mathrm{wt} \%$ of a-FeOOH nano-wires has larger diameter and also for $40 \%$ higher value of Young's modulus than that for composition containing $2.5 \mathrm{wt} \%$ of a-FeOOH nanowires. There is also no reason why the nanoparticle additive should influence the strength of interfiber contacts or directional arrangement of fibers in the mat so observed increase in the mechani-cal properties can be attributed solely to the inherent rein-forcing effect of goethite nanowires.

\section{CONCLUSIONS}

We introduced a-FeOOH nanowires synthesized by straightforward and environmentally friendly "chimie douce" method into aqueous PVA solution my simple mixing. The obtained homogeneous dispersions were electrospun into fiber mats containing up to $10 \mathrm{wt} \%$ of goethite nanowires aligned along the fiber axis. The presence of crystalline goethite in the material was confirmed by TEM and XRD, but ATR-FTIR and NMR revealed no noticeable changes in the crystallinity of the polymer or covalent bonding to the mineral additive. The melting temperature was found to be unaffected by the concentration of the additive by DSC analysis, however the glass transition temperature increased by 12 degrees. These findings indicate the formation of glassy interphase regions around the particle inclusions. Tensile tests were consistent with the latter as PVA with $10 \mathrm{wt} \%$ goethite exhibited an outstanding fivefold increase in the elastic modulus and 2.5-fold improvement of tensile strength in comparison with mats from neat PVA. We suggest that the a-FeOOH is grafted with PVA molecules in the precursor solution through hydrogen bonding, possibly also complexation with $\mathrm{Fe}^{31}$. Further entanglements of adsorbed PVA chains with the surrounding matrix polymer in obtained composite lead to the formation of extensive interphase that mediates stress transfer from the matrix to the additive particles during mechanical defor-mation. The saturation of observed influence of nanowire additive suggests $10 \mathrm{wt} \%$ may be sufficient for achieving continuity of interphase between the aligned additive par-ticles throughout the material. Developed method presents an attractive approach to eco-friendly functional biopoly-mers as significant improvement of mechanical properties of electrospun PVA is achieved by combining materials from natural sources with sustainable processing methods.

\section{ACKNOWLEDGMENTS}

The authors kindly acknowledge the financial support of the Estonian Research Council for the post-doctoral research grants of personal research funding in projects PUT1096 and PUTJD578 as well as Institutional Research Funding Projects, IUT20-17, and IUT23-7.

\section{REFERENCES}

1. S.S. Gglin and M.D. Gurol, Environ. Sci. Technol., 32, 1417 (1998).

2. A.H. Barber, D. Lu, and N.M. Pugno, J. R. Soc. Interface, 12, 20141326 (2015).

3. D. Chicot, J. Mendoza, A. Zaoui, G. Louis, V. Lepingle, F. Roudet, and J. Lesage, Mater. Chem. Phys., 129, 862 (2011).

4. L.A. Wijenayaka, G. Rubasinghege, J. Baltrusaitis, and V.H. Grassian, J. Phys. Chem. C, 116, 12566 (2012).

5. M. Peng, G. Xiao, X. Tang, and Y. Zhou, Macromolecules, 47, 8411 (2014).

6. P. Song, Z. Xu, Y. Lu, and Q. Guo, Macromolecules, 48, 3975 (2015).

7. P. Song, Z. Xu, and Q. Guo, ACS Macro Lett., 2, 1100 (2013).

8. A. Gajovica, $\quad$ A.M.T. Silvab, R.A. Segundob, S. Sturmc, B. Jancarc, ${ }^{\circ}$ and M. Ceh, Appl. Catal. B, 103, 351 (2011).

9. H.F. Chen, G.D. Wei, X. Han, S. Li, P.P. Wang, M. Chubik, A. Gromov, Z.P. Wang, and W. Han, J. Mater. Sci: Mater. Electron., 22, 252 (2011).

10. N. Chemin, L. Rozes, C. Chaneac, S. Cassaignon, E. Le Bourhis, J.P. Jolivet, O. Spalla, E. Barthel, and C. Sanchez, Chem. Mater., 20, 4602 (2008).

11. P. Supaphol and S. Chuangchote, J. Appl. Polym. Sci., 131, 969 (2014).

12. X.H. Qin and S.Y. Wang, J. Appl. Polym. Sci., 102, 1285 (2006).

13. B. Bolto, T. Tran, M. Hoang, and Z. Xie, Prog. Polym. Sci., 34, 969 (2009).

14. X. Tang and S. Alavi, Carbohyd. Polym., 85, 7 (2011).

15. S. Moulay, Polym.-Plast. Technol., 54, 1289 (2015). 
16. D. Lopez, I. Cendoya, F. Torres, J. Tejada, and C. Mijangos, J. Appl. Polymer Sci., 82, 3215 (2011).

17. L. Chen, K. Zheng, X. Tian, K. Hu, R. Wang, C. Liu, Y. Li, and P. Cui, Macromolecules, 43, 1076 (2010).

18. Y. Liu and S. Kumar, ACS Appl. Mater. Interfaces, 6, 6069 (2014).

19. H.K.F. Cheng, N.G. Sahoo, Y.P. Tan, Y. Pan, H. Bao, L. Li, S.H. Chan, and J. Zhao, ACS Appl. Mater. Interfaces, 4, 2387 (2012).

20. W. Li, J. Yue, and S. Liu, Ultrason. Sonochem, 19, 479 (2012).

21. A. Pakzad, J. Simonsen, and R.S. Yassar, Nanotechnology, 23, 085706 (2012)

22. A. Sutka, A. Sutka, S. Gaidukov, M. Timusk, J. Gravitis, and S. Kukle, Holzforschung, 69, 737 (2015).

23. B.K. Tan, Y.C. Ching, S.C. Poh, L.C. Abdullah, and S.N. Gan, Polymer, 7, 2205 (2015).

24. Z.H. Mbhele, M.G. Salemane, C.G.C.E. van Sittert, J.M. Nedeljkovic', V. Djokovic', and A.S. Luyt, Chem. Mater., 15, 5019 (2003).

25. C.M. Paranhos, B.G. Soares, R.N. Oliveira, and L.A. Pessan, Macromol. Mater. Eng., 292, 620 (2007).

26. J. Moczo, E. Fekete, and B. Pukanszky, J. Adhesion, 78, 861 (2002).

27. B. Natarajan, Y. Li, H. Deng, L.C. Brinson, and L.S. Schadler, Macromolecules, 46, 2833 (2013).

28. D. Brown, P. Mele, S. Marceau, and N.D. Alberola, Macromolecules, 36, 1395 (2003).

29. S. Merabia, P. Sotta, and D.R. Long, Macromolecules, 41, 8252 (2008)

30. A. Karatrantos, N. Clarke, R.J. Composto, and K.I. Winey, Soft Matter, 12, 2567 (2016).

31. J. Choi, H. Shin, S. Yang, and M. Cho, Compos. Struct. 119, 365 (2015).

32. Y. Gao, J. Liu, J. Shen, L. Zhang, and D. Cao, Polymer, 55, 1273 (2014)

33. C. Pastorino, K. Binder, T. Kreer, and M. M€uller, J. Chem. Phys., 124, 064902 (2006).

34. R. Toth, F. Santese, S.P. Pereira, D.R. Nieto, S. Pricl, M. Fermeglia, and P. Posocco, J. Mater. Chem., 22, 5398 (2012).

35. V. Padmanabhan, J. Chem. Phys., 139, 144904 (2013).

36. P. Rittigstein, R.D. Priestley, L.J. Broadbelt, and J.M. Torkelson, Nat. Mater., 6, 278 (2007).

37. V. Ganesan and A. Jayaraman, Soft Matter., 10, 13 (2014).

38. Y. Gao, J. Liu, J. Shen, Y. Wu, and L. Zhang, Phys. Chem. Chem. Phys, 16, 21372 (2014).

39. E. Masnada, S. Merabia, M. Couty, and J.L. Barrat, Soft Matter, 9, 10532 (2013).

40. R.D. Peng, H.W. Zhou, H.W. Wang, and L. Mishnaevsky, Jr., Comp. Mater. Sci., 60, 19 (2012).
41. V. Marcadon, D. Brown, E. Herve, P. Mele, N.D. Alberola, and A. Zaoui, Comp. Mater. Sci., 79, 495 (2013).

42. S.K. Kumar, and R. Krishnamoorti, Annu. Rev. Chem. Biomol. Eng., 1, 37 (2010).

43. Y. Dou, K. Lin, and J. Chang, Nanoscale, 3, 1508 (2011).

44. Y. Dou, C. Wu, and J. Chang, Acta Biomater., 8, 4139 (2012).

45. J.G. Chen, X.H. Li, W.G. Cui, C.Y. Xie, J. Zou, and B. Zou, Adv. Eng. Mater., 12, B529 (2010).

46. B.V. Kavanagh, A.M. Posner, and J.P. Quirk, J. Soil. Sci., 27, 467 (1976).

47. S. Chen, G. Schueneman, R.B. Pipes, J. Youngblood, and R.J. Moon, Biomacromolecules, 15, 3827 (2014).

48. M. Bashouti, W. Salalha, M. Brumer, E. Zussman, and E. Lifshitz, Chem. Phys. Chem., 7, 102 (2006).

49. X. Zhou, H. Yang, C. Wang, X. Mao, Y. Wang, Y. Yang, and G. Liu, J. Phys. Chem. C, 114, 17051 (2010).

50. J.P. Jolivet, C. Chaneac, and E. Tronc, Chem. Commun., 10, 481 (2004).

51. D.H. Reneker, and A.L. Yarin, Polymer, 49, 2387 (2008).

52. J. Feng, X. Ma, H. Mao, B. Liu, and X. Zhao, J. Mater. Res., 26, 2691 (2011).

53. R. Frost, H.Y. Zhu, P. Wu, and T. Bostrom, Mater. Lett., 59, 2238 (2005).

54. A. Omoike, and J. Chorover, Geochim. Cosmo. Acta., 70, 827 (2006).

55. E.J. Elzinga, and D.L. Sparks, J. Colloid Interface Sci., 308, 53 (2007).

56. C. Luengo, M. Brigante, J. Antelo, and M. Avena, J. Col-loid Interface Sci., 300, 511 (2006).

57. M.I. Tejedor-Tejedor, and M.A. Anderson, Langmuir, 6, 602 (1990).

58. P. Persson, N. Nilsson, and S. Sj€oberg, J. Colloid Interface Sci., 177, 263 (1996).

59. M. Kobayashi, I. Ando, T. Ishii, and S. Amiya, J. Mol. Struct., 440, 155 (1998).

60. K. Masuda, H. Kaji, and F. Horii, J. Polym. Sci. B-Polym. Phys., 38, 1 (2000).

61. A. Bansal, H. Yang, C. Li, K. Cho, B.C. Benicewicz, S.K. Kumar, and L.S. Schadler, Nat. Mater., 4, 693 (2005).

62. H. Oh and P.F. Green, Nat. Mat., 8, 139 (2009).

63. L. Meli, A. Arceo, and P.F. Green, Soft Matter., 5, 533 (2009).

64. J.O. Zoppe, M.S. Peresin, Y. Habibi, R.A. Venditti, and O.J. Rojas, Appl. Mater. Interfaces, 1, 1996 (2009).

65. U. Stachewicz, R.J. Bailey, W. Wang, and A.H. Barber, Polymer, 53, 5132 (2012).

66. Q. Fu, Y. Jin, X. Song, J. Gao, X. Han, X. Jiang, Q. Zhao, and D. Yu, Nanotechnology, 21, 095703 (2010). 